\title{
ON LEFT AND RIGHT BROWDER OPERATORS
}

\author{
Snežana Č. Živković-Zlatanović, Dragan S. Djordjević, \\ AND ROBIN E. HARTE
}

\begin{abstract}
We discuss the perturbation theory of "left" and "right" Browder operators, which come somewhere between Browder operators and semi Browder operators.
\end{abstract}

\section{Introduction}

Let $X$ and $Y$ be infinite dimensional Banach spaces, and let $B L(X, Y)$ be the set of all linear bounded operators from $X$ to $Y$. Recall $[4,11]$ that $T \in$ $B L(X, Y)$ is Fredholm if and only if it has finite dimensional null space and closed range of finite codimension, in which case

$$
\operatorname{index}(T)=\operatorname{nul}(T)-\operatorname{def}(T)=\operatorname{dim} T^{-1}(0)-\operatorname{dim}(Y / T X) .
$$

When $\operatorname{index}(T)=0$ we say that $T \in B L(X, Y)$ is Weyl. Necessary and sufficient for $T \in B L(X, Y)$ to be Fredholm is that it be essentially invertible, in the sense that there are $T^{\prime}, T^{\prime \prime}$ in $B L(Y, X)$ for which

$$
I-T^{\prime} T \in J(X), I-T T^{\prime \prime} \in J(Y)
$$

where $J$ can be either the finite rank operators or the compact operators or indeed the inessential operators - all three conditions are equivalent. Since everything in the finite rank operators has generalized inverses it follows that

$$
T \text { Fredholm } \Longrightarrow T \text { relatively regular. }
$$

It then follows that if $(0.2)$ holds it can be arranged that $T^{\prime}=T^{\prime \prime}$ is a generalized inverse for $T$.

The Weyl operators can be distinguished among the Fredholm operators as those for which

$$
T \in B L(X, Y)^{-1}+J(X, Y),
$$

where again $J$ can be finite rank, compact or inessential; it is also necessary and sufficient that $T$ is Fredholm and has an invertible generalized inverse.

Received June 23, 2010; Revised November 3, 2010.

2010 Mathematics Subject Classification. 47A53, 47 A55.

Key words and phrases. semi Browder, Riesz perturbations.

The work is supported by the Ministry of Science of Serbia, grant no. 174007 .

(C)2011 The Korean Mathematical Society 
More general than the Fredholm operators are the left and right Fredholm operators, in which only half the condition (0.2) holds; more general still are the upper and lower semi Fredholm operators, in which either the null space or the quotient of the range are finite dimensional. We shall also describe as upper semi Weyl, or left Weyl, an upper semi Fredholm, or left Fredholm, operator of non-positive index, and as lower semi Weyl, or right Weyl, a lower semi Fredholm, or right Fredholm, operator of non-negative index. The set of all upper (lower) semi Fredholm operators on $X$ is denoted by $\Phi_{+}(X)\left(\Phi_{-}(X)\right)$. Let $P\left(\Phi_{+}(X)\right)$ denote the perturbation class of $\Phi_{+}(X)$, i.e., $P\left(\Phi_{+}(X)\right)=$ $\left\{A \in B L(X): A+T \in \Phi_{+}(X)\right.$ for every $\left.T \in \Phi_{+}(X)\right\}$. Analogously we define $P\left(\Phi_{-}(X)\right)$.

When in particular $Y=X$ we meet Browder operators, which are Fredholm of finite ascent and descent: there is $n \in \mathbf{N}$ for which

$$
T^{-n-1}(0) \subseteq T^{-n}(0) \text { and } T^{n} X \subseteq T^{n+1}(X) ;
$$

necessarily such operators are of index zero, therefore "Weyl". In this note we look at left, right and semi Browder operators:

Definition 1. $T \in B L(X) \equiv B L(X, X)$ will be called upper semi Browder if and only if it is upper semi Fredholm of finite ascent, and lower semi Browder if and only if it is lower semi Fredholm of finite descent. We shall say that $T \in B L(X)$ is left Browder if and only if it is left Fredholm of finite ascent, and right Browder if and only if it is right Fredholm of finite descent.

Each of these four sets of operators is open; evidently

(1.1) $T$ left Browder $\Longleftrightarrow T$ upper semi Browder and relatively regular,

(1.2) $T$ right Browder $\Longleftrightarrow T$ lower semi Browder and relatively regular, and we have ([9, Proposition 38.5])

$$
T \text { left Browder } \Longrightarrow T \text { left Weyl } \Longrightarrow T \text { left Fredholm }
$$

and

$$
T \text { right Browder } \Longrightarrow T \text { right Weyl } \Longrightarrow T \text { right Fredholm. }
$$

An operator which is both upper and lower semi Browder is Browder. It is also true, for each of these four "non singularities", that

$$
S T=T S \Longrightarrow(S, T \text { non singular } \Longleftrightarrow S T \text { non singular }) \text {. }
$$

It follows in particular that if $T \in B L(X)$ is in one of these classes, then so are all powers $T^{n}$. Since semi Browder operators have closed range it follows that for semi Browder operators

$$
T^{\infty}(X) \equiv \bigcap_{n=1}^{\infty} T^{n}(X) \text { is closed. }
$$


Fixing the operator $T \in B L(X)$, we shall write $S^{\sim} \in B L\left(T^{\infty}(X)\right)$ for the restriction of an operator $S \in B L(X)$ which leaves the hyperrange invariant, in particular for $S \in \operatorname{comm}(T)$.

The perturbation theory of upper and lower semi Browder operators has been worked out. We shall write

$$
\operatorname{comm}(T)=\{S \in B L(X): S T=T S\}
$$

for the commutant of $T \in B L(X)$,

$$
\operatorname{comm}^{-1}(T)=\operatorname{comm}(T)_{\cap} B L(X)^{-1}
$$

for the "invertible commutant" of $T$, and if $\delta>0$

$$
\operatorname{comm}_{\delta}^{-1}(T)=\left\{S \in \operatorname{comm}^{-1}(T):\|S\|<\delta\right\} .
$$

We write Nil $B L(X)$ and QNil $B L(X)$ for the nilpotent and the quasinilpotent operators in $B L(X)$, we write $B_{00}(X), B_{0}(X)$ and $B_{00}^{\sim}(X)$ for the finite rank, compact and inessential operators on $X$, and finally $B L(X)^{+}, B L(X)^{-}$, $B L(X)_{\text {left }}^{-1}$ and $B L(X)_{\text {right }}^{-1}$ for the bounded below, the onto, the left and the right invertible operators on $X$. We also recall the "Kato decomposition":

Definition 2. We shall say that an operator $T \in B L(X)$ is almost invertible provided

$$
\exists \delta>0: 0<|\lambda|<\delta \Longrightarrow T-\lambda I \in B L(X)^{-1},
$$

that it is a Kato operator provided

$$
T^{-1}(0) \subseteq T^{\infty}(X) \text { and } T X=\operatorname{cl}(T X),
$$

and that it is essentially Kato provided there is a projection $P=P^{2} \in \operatorname{comm}(T)$ for which, with $X_{1}=P^{-1}(0), X_{0}=P(X)$ and $T_{j}: X_{j} \rightarrow X_{j}$ the restrictions,

$$
P \in B_{00}(X) ; T_{0} \in \text { Nil } B L\left(X_{0}\right) ; T_{1} \text { Kato on } X_{1} \text {. }
$$

(2.3) is also known as a Kato decomposition for $T$. Kato operators are sometimes said [5] to be Kato non singular; a Kato operator with a generalized inverse is known as a Saphar operator, or alternatively [5] said to be Kato invertible. We shall extend the terminology of (2.1) to other relevant non singularities such as bounded below and onto. With a little bit of gap theory, Müller shows ([11, Theorem 12.11]) that if $T \in B L(X)$, then $T$ is almost invertible Kato if and only if it is invertible, and more generally ([11, Corollary 12.4])

$$
T \text { almost bounded below Kato } \Longrightarrow T \text { bounded below, }
$$

$$
T \text { almost onto Kato } \Longrightarrow T \text { onto. }
$$

Kato operators satisfy ([11, Theorem 12.2$])$ the condition $(1.6)$, and ([11, Theorem 12.15]) also

$$
T T^{\infty}(X)=T^{\infty}(X)
$$


We recall ([14, Theorem 2.1]; [11, Theorem 16.21]) that semi Fredholm operators are essentially Kato. We remark also that if $P=P^{2}$ commutes with $T \in B L(X)$, then, with again $X_{1}=P^{-1}(0), X_{0}=P(X)$ and $T_{j}: X_{j} \rightarrow X_{j}$ the restrictions,

$$
T \in T B L(X) T \Longleftrightarrow\left(T_{1} \in T_{1} B L\left(X_{1}\right) T_{1} \text { and } T_{0} \in T_{0} B L\left(X_{0}\right) T_{0}\right),
$$

and that

(2.8) $T(X)=\operatorname{cl} T(X) \Longleftrightarrow\left(T_{1}\left(X_{1}\right)=\operatorname{cl} T_{1}\left(X_{1}\right)\right.$ and $\left.T_{0}\left(X_{0}\right)=\operatorname{cl} T_{0}\left(X_{0}\right)\right)$ :

this is easily checked by writing out $2 \times 2$ operator matrices.

Theorem 3. If $T \in B L(X)$, then the following are equivalent:

\section{$T$ upper semi Browder;}

(3.2) T upper semi Fredholm and $\exists \delta>0: T-\operatorname{comm}_{\delta}^{-1}(T) \subseteq B L(X)^{+}$;

\section{$T$ upper semi Fredholm and almost bounded below;}

(3.4) $\exists P=P^{2} \in B_{00}(X)_{\cap} \operatorname{comm}(T): T_{0} \in \operatorname{Nil} B L\left(X_{0}\right), T_{1} \in B L\left(X_{1}\right)^{+}$;

(3.5) $\exists P=P^{2} \in B_{00}(X)_{\cap} \operatorname{comm}(T): T P \in \operatorname{Nil} B L(X), T+P \in B L(X)^{+}$;

$$
\begin{aligned}
& \exists K \in B_{00}(X)_{\cap} \operatorname{comm}(T): T-K \in B L(X)^{+} ; \\
& \exists K \in B_{0}(X)_{\cap} \operatorname{comm}(T): T-K \in B L(X)^{+} .
\end{aligned}
$$

Proof. If $S \in \mathrm{comm}^{-1}(T)$ is invertible and commutes with $T$, then

$$
(T-S)^{\sim-1}(0)=(T-S)^{-1}(0) \subseteq T^{\infty}(X) .
$$

If $\operatorname{dim} T^{-1}(0)<\infty$, then, as in $(2.6)$,

$$
T\left(T^{\infty} X\right)=T^{\infty}(X) .
$$

There is therefore $\delta>0$ for which $\left(\left[5\right.\right.$, Theorem 5]), if $S \in \operatorname{comm}_{\delta}^{-1}(T)$,

$$
\begin{aligned}
\operatorname{dim}(T-S)^{-1}(0) & =\operatorname{dim}(T-S)^{\sim-1}(0)=\operatorname{index}(T-S)^{\sim} \\
& =\operatorname{index}\left(T^{\sim}\right)=\operatorname{dim} T^{\sim-1}(0)<\infty:
\end{aligned}
$$

the second equality is because $(T-S)^{\sim}$ is onto for small $\|S\|$, and the third is index continuity for lower semi Fredholm operators ([11, Theorem 16.17, Corollary 20.2]) on $T^{\infty}(X)$. Now ([4, Theorem 7.8.2])

$$
\operatorname{asc}(T)<\infty \Longrightarrow T^{-1}(0)_{\cap} T^{\infty}(X)=\{0\} \Longleftrightarrow T^{\sim-1}(0)=\{0\} .
$$

It follows that $T-S$ is upper semi Fredholm and one one, therefore bounded below: $(3.1) \Longrightarrow(3.2) \Longrightarrow(3.3)$. Towards implication $(3.3) \Longrightarrow(3.4)$ take the projection $P=P^{2}$ from the Kato decomposition (2.1). Since $T_{1}$ is Kato and almost bounded below it follows from (2.4) that $T_{1}$ is bounded below, while $T_{0}$ is nilpotent. Implication $(3.4) \Longrightarrow(3.5)$ is $2 \times 2$ matrices again ([10, Theorem 4.2]). For implication $(3.5) \Longrightarrow(3.6) \Longrightarrow(3.7)$ define $K=-P$. Finally if 
$K \in B_{0}(X)$ is compact and commutes with bounded below $T-K$, then ([4, Theorem 7.9.2]) asc $((T-K)+K)<\infty$.

We shall see below (Theorem 7) that the condition (3.6) can be improved ([15, Corollary 2]) to inessential, and indeed Riesz, perturbations:

$$
\exists K \in B_{00}^{\sim}(X)_{\cap} \operatorname{comm}(T): T-K \in B L(X)^{+} .
$$

Theorem 4. If $T \in B L(X)$, then the following are equivalent:

T lower semi Browder;

(4.2) T lower semi Fredholm and $\exists \delta>0: T-\operatorname{comm}_{\delta}^{-1}(T) \subseteq B L(X)^{-}$;

T lower semi Fredholm and almost onto;

(4.4) $\exists P=P^{2} \in B_{00}(X)_{\cap} \operatorname{comm}(T): T_{0} \in \operatorname{Nil} B L\left(X_{0}\right), T_{1} \in B L\left(X_{1}\right)^{-}$;

(4.5) $\exists P=P^{2} \in B_{00}(X)_{\cap} \operatorname{comm}(T): T P \in \operatorname{Nil} B L(X), T+P \in B L(X)^{-}$;

$$
\begin{aligned}
& \exists K \in B_{00}(X)_{\cap} \operatorname{comm}(T): T-K \in B L(X)^{-} ; \\
& \exists K \in B_{0}(X)_{\cap} \operatorname{comm}(T): T-K \in B L(X)^{-} .
\end{aligned}
$$

Proof. Implication (4.1) $\Longrightarrow(4.2) \Longrightarrow(4.3)$ follows from the same punctured neighbourhood argument as for Theorem 3, and then (4.4) is again the Kato decomposition: since $T_{1}$ is Kato and almost onto it follows from (2.5) that $T_{1}$ is onto, while $T_{0}$ is again nilpotent. Finally if $K \in B_{0}(X)$ is compact and commutes with onto $T-K$, then $([4$, Theorem 7.9.2]) $\operatorname{dsc}((T-K)+K)<$ $\infty$.

Theorem 5. If $T \in B L(X)$, then the following are equivalent:

$$
T \text { left Browder; }
$$

$$
T \text { left Fredholm and } \exists \delta>0: T-\operatorname{comm}_{\delta}^{-1}(T) \subseteq B L(X)_{\text {left }}^{-1} \text {; }
$$

$$
\exists P=P^{2} \in B_{00}(X)_{\cap} \operatorname{comm}(T): T P \in \operatorname{Nil} B L(X), T+P \in B L(X)_{l e f t}^{-1} ;
$$

$$
\begin{aligned}
& \exists K \in B_{00}(X)_{\cap} \operatorname{comm}(T): T-K \in B L(X)_{\text {left }}^{-1} ; \\
& \exists K \in B_{0}(X)_{\cap} \operatorname{comm}(T): T-K \in B L(X)_{\text {left }}^{-1} .
\end{aligned}
$$


Proof. Much of Theorem 5 is a consequence of Theorem 3: if $T \in B L(X)$ is left Browder, then (3.2) holds, and hence also (5.2), since now each $T-S$ is both left Fredholm and one one. A similar comment applies to (5.5): $T_{1}$ is by (3.4) bounded below and by (2.7) relatively regular. Finally if $K \in B_{0}(X)$ is compact and commutes with left invertible $T-K$, then $T=(T-K)+K$ is left Fredholm of finite ascent.

Theorem 6. If $T \in B L(X)$, then the following are equivalent:

$$
\text { T right Browder; }
$$

$$
T \text { right Fredholm and } \exists \delta>0: T-\operatorname{comm}_{\delta}^{-1}(T) \subseteq B L(X)_{\text {right }}^{-1} \text {; }
$$

$$
T \text { right Fredholm and almost right invertible; }
$$

(6.5) $\exists P=P^{2} \in B_{00}(X)_{\cap} \operatorname{comm}(T): T_{0} \in \mathrm{Nil} B L\left(X_{0}\right), T_{1} \in B L\left(X_{1}\right)_{\text {right }}^{-1}$;

$$
\exists P=P^{2} \in B_{00}(X)_{\cap} \operatorname{comm}(T): T P \in \operatorname{Nil} B L(X), T+P \in B L(X)_{\text {right }}^{-1} ;
$$

$$
\begin{aligned}
& \exists K \in B_{00}(X)_{\cap} \operatorname{comm}(T): T-K \in B L(X)_{\text {right }}^{-1} ; \\
& \exists K \in B_{0}(X)_{\cap} \operatorname{comm}(T): T-K \in B L(X)_{\text {right }}^{-1} .
\end{aligned}
$$

Proof. Much of Theorem 6 is a consequence of Theorem 4: if $T \in B L(X)$ is right Browder, then (4.2) holds, and hence also (6.2), since now each $T-S$ is both right Fredholm and onto. A similar comment applies to (6.5): $T_{1}$ is by (4.4) onto and by (2.7) relatively regular. Finally if $K \in B_{0}(X)$ is compact and commutes with right invertible $T-K$, then $T=(T-K)+K$ is right Fredholm of finite descent.

If we introduce Fredholm, Weyl and Browder spectra $\omega \in\left\{\sigma_{e}, \sigma_{w}, \sigma_{b}\right\}$ in the obvious way, we shall also write

$$
\omega^{+}, \omega^{-}, \omega^{\text {left }}, \omega^{\text {right }}
$$

for the corresponding upper, lower, left and right versions. We recall ([4, Theorem 9.8.3]; [11, Corollary 20.20])

$$
\sigma_{e}(T) \subseteq \sigma_{w}(T) \subseteq \sigma_{b}(T)=\sigma_{e}(T) \cup \operatorname{acc} \sigma(T) .
$$

This has upper, lower, left and right versions: the conditions (3.3)-(6.3) guarantee respectively the upper semi, lower semi, left and right analogues of (6.9). Each of the semi Browder conditions of Definition 1 are, more generally, stable under commuting Riesz perturbations: 
Theorem 7. If $T \in B L(X)$ and $K \in B L(X)$ is Riesz, then

$$
\begin{gathered}
T K=K T \Longrightarrow \sigma_{b}^{+}(T+K)=\sigma_{b}^{+}(T), \\
T K=K T \Longrightarrow \sigma_{b}^{-}(T+K)=\sigma_{b}^{-}(T), \\
T K=K T \Longrightarrow \sigma_{b}^{\text {left }}(T+K)=\sigma_{b}^{\text {left }}(T)
\end{gathered}
$$

and

$$
T K=K T \Longrightarrow \sigma_{b}^{r i g h t}(T+K)=\sigma_{b}^{r i g h t}(T) .
$$

Proof. Towards (7.3) we claim that if $\lambda \in \mathbf{C}$ is arbitrary

$$
\sigma_{e}^{\text {left }}(T+\lambda K)=\sigma_{e}^{\text {left }}(T)
$$

and

$$
\operatorname{acc} \sigma^{l e f t}(T+\lambda K)=\operatorname{acc} \sigma^{l e f t}(T) .
$$

For (7.5) combine the two variable spectral mapping theorem for the left spectrum ([3]; [4]; [11, Theorem 8.8]) with the fact that in the Calkin algebra $B L(X) / B_{0}(X)$ the coset $k=K+B_{0}(X)$ is quasinilpotent:

$\sigma^{l e f t}(t+\lambda k)=\left\{\alpha+\lambda \beta:(\alpha, \beta) \in \sigma^{\text {left }}(t, k)\right\} \subseteq \sigma^{\text {left }}(t)+\lambda \sigma^{\text {left }}(k)=\sigma^{\text {left }}(t)$,

since $\sigma(k)=\{0\}$, and the reverse inclusion follows from $t=(t+k)+(-k)$. Towards (7.6) we have, using this time the two variable spectral mapping theorem in the algebra of operators,

$$
\sigma^{l e f t}(T+\lambda K)=\left\{\alpha+\lambda \beta:(\alpha, \beta) \in \sigma^{l e f t}(T, K)\right\} \subseteq \sigma^{l e f t}(T)+\lambda \sigma^{l e f t}(K),
$$

and $([6$, Theorem 6$])$ simple properties of accumulation points,

$$
\begin{aligned}
\operatorname{acc} \sigma^{l e f t}(T+\lambda K) & \subseteq \operatorname{acc}\left(\sigma^{l e f t}(T)+\lambda \sigma^{l e f t}(K)\right) \\
& \subseteq \operatorname{acc} \sigma^{l e f t}(T)+\lambda \operatorname{acc} \sigma^{l e f t}(K)=\operatorname{acc} \sigma^{l e f t}(T) .
\end{aligned}
$$

This gives (7.3). For (7.4) replace the left spectrum by the right. For (7.1) and (7.2) pass to the "essential enlargement" [1], and ([11, Theorem 17.6, Theorem 17.9]) replace the left spectrum by the approximate point and the defect spectrum of the essential enlargements of $T, K$ and $(T, K)$.

We remark that it is sufficient for (7.5) that the cosets $t, k$ commute in the Calkin algebra $B L(X) / B_{0}(X)$, and that this remains sufficient if the Calkin algebra is replaced by the quotient $B L(X) / B_{00}^{\sim}(X)$. As we remarked above (3.8), Theorem 7 has the effect of extending each of the conditions (3.7), (4.7), (5.8) and (6.8) from compact to Riesz operators; the proof we have given for it also tells us something about left and right Weyl operators: 
Theorem 8. If $K \in B L(X)$, then the following are equivalent:

K Riesz;

$$
\begin{gathered}
T K-K T \in B_{00}^{\sim}(X) \Longrightarrow \sigma_{e}^{\text {left }}(T)=\sigma_{e}^{\text {left }}(T+K) ; \\
T K-K T \in B_{00}^{\sim}(X) \Longrightarrow \sigma_{e}^{\text {right }}(T)=\sigma_{e}^{\text {right }}(T+K) ; \\
T K-K T \in B_{00}^{\sim}(X) \Longrightarrow \sigma_{w}^{l e f t}(T)=\sigma_{w}^{l e f t}(T+K) ; \\
T K-K T \in B_{00}^{\sim}(X) \Longrightarrow \sigma_{w}^{\text {right }}(T)=\sigma_{w}^{\text {right }}(T+K) ; \\
T \in \operatorname{comm}(K) \Longrightarrow \sigma_{e}^{\text {left }}(T)=\sigma_{e}^{l e f t}(T+K) ; \\
T \in \operatorname{comm}(K) \Longrightarrow \sigma_{e}^{\text {right }}(T)=\sigma_{e}^{\text {right }}(T+K) ; \\
T \in \operatorname{comm}(K) \Longrightarrow \sigma_{w}^{\text {left }}(T)=\sigma_{w}^{\text {left }}(T+K) ; \\
T \in \operatorname{comm}(K) \Longrightarrow \sigma_{w}^{\text {right }}(T)=\sigma_{w}^{\text {right }}(T+K) .
\end{gathered}
$$

Proof. If (8.1) holds, then (8.2), and hence also (8.6), follows from (7.7) with $\lambda=1$, and similarly (8.3) and (8.7); now the continuity of the index gives also (8.4) and (8.5), and hence also (8.8) and (8.9). Conversely taking $T=O$ shows that each of (8.6)-(8.9) imply that $K \in B L(X)$ is Riesz.

From Theorem 8 it follows that if $T K-K T$ is "inessential" and $K$ is Riesz, then the left and right Fredholm and Weyl properties are transmitted from $T$ to $T+K$. It also follows from Theorem 7 and Theorem 8 that if $K$ is Riesz and commutes with $T$ and if "Browder's theorem holds" for $T$, then it also holds for $T+K$. This is the common generalization of the two parts of Theorem 11 of $[7]$.

It is possible to use [16, Theorem 30] to prove the following: if $K$ is Riesz and $T K=K T$, then $\sigma_{w}^{+}(T+K)=\sigma_{w}^{+}(T)$ and $\sigma_{w}^{-}(T+K)=\sigma_{w}^{-}(T)$. This is the method applied in [2, Theorem 4.1]. A generalization of that result from [16] is presented in [17, Corollary 1]. Precisely, the condition " $T K-K T$ is compact" in [16] is replaced with " $T K-K T$ is in the perturbation class of the set of all upper semi-Fredholm operators" in [17]. Therefore, if $K$ is Riesz, then for every $T$ which is upper (lower) semi Fredholm and satisfies that $T K-K T \in P\left(\Phi_{+}(X)\right)$ (or $P\left(\Phi_{-}(X)\right)$ ) it follows that $T+K$ is upper (lower) semi Fredholm and index $(\mathrm{T}+\mathrm{K})=\operatorname{index}(\mathrm{T})([17$, Corollary 2$])$. Thus the following statements are equivalent:

$$
\begin{aligned}
& K \text { is Riesz; } \\
& T K-K T \in P\left(\Phi_{+}(X)\right) \Longrightarrow \sigma_{e}^{+}(T)=\sigma_{e}^{+}(T+K) ; \\
& T K-K T \in P\left(\Phi_{-}(X)\right) \Longrightarrow \sigma_{e}^{-}(T)=\sigma_{e}^{-}(T+K) ; \\
& T K-K T \in P\left(\Phi_{+}(X)\right) \Longrightarrow \sigma_{w}^{+}(T)=\sigma_{w}^{+}(T+K) ; \\
& T K-K T \in P\left(\Phi_{-}(X)\right) \Longrightarrow \sigma_{w}^{-}(T)=\sigma_{w}^{-}(T+K) ; \\
& T \in \operatorname{comm}(K) \Longrightarrow \sigma_{e}^{+}(T)=\sigma_{e}^{+}(T+K) ;
\end{aligned}
$$




$$
\begin{aligned}
& T \in \operatorname{comm}(K) \Longrightarrow \sigma_{e}^{-}(T)=\sigma_{e}^{-}(T+K) \\
& T \in \operatorname{comm}(K) \Longrightarrow \sigma_{w}^{+}(T)=\sigma_{w}^{+}(T+K) \\
& T \in \operatorname{comm}(K) \Longrightarrow \sigma_{w}^{-}(T)=\sigma_{w}^{-}(T+K)
\end{aligned}
$$

It is familiar that the Weyl and the Browder spectrum of an operator can be written as the intersection of the spectrums of its compact, and its commuting compact, perturbations. Theorem 8 extends this to upper and lower, and left and right spectra, and Riesz perturbations:

Theorem 9. If $T \in B L(X)$, then, for each $*=$ left, right, and $J(X)$ any non zero ideal of Riesz operators,

$$
\sigma_{w}^{*}(T)=\bigcap\left\{\sigma^{*}(T+K): K \text { Riesz, } T K-K T \in J(X)\right\}
$$

and, for each for each $*=+,-$, left, right,

$$
\sigma_{b}^{*}(T)=\bigcap\left\{\sigma^{*}(T+K): K \text { Riesz commutes with } T\right\} .
$$

Also, for each $*=$ left, right,

$$
\sigma_{b}^{*}(T)=\sigma_{w}^{*}(T) \cup \bigcap\left\{\pi^{*}(T+K): K \text { Riesz commutes with } T\right\},
$$

where $\pi^{\text {left }}$ and $\pi^{\text {right }}$ are the point spectrum and the point spectrum of the dual.

Proof. Inclusion one way in (9.1) follows from (8.4) and (8.5), and in (9.2) follows from Theorem 7 . In the other direction ([11, Theorem 19.7])

$$
\sigma_{w}^{*}(T)=\bigcap\left\{\sigma^{*}(T+K): K \in J(X)\right\}
$$

and ([11, Theorem 20.21])

$$
\sigma_{b}^{*}(T)=\bigcap\left\{\sigma^{*}(T+K): K \in J(X)_{\cap} \operatorname{comm}(T)\right\} .
$$

For (9.3), combine these with equality

$$
\sigma^{*}(T)=\sigma_{w}^{*}(T) \cup \pi^{*}(T) .
$$

Rakočević ([12, Corollary 3.2]) proved the analogue of (9.3) for the Browder and the Weyl spectrum. The boundary of the Browder spectrum is a subset of the essential spectrum:

Theorem 10. If $T \in B L(X)$, then for each $*=+,-$, left, right there is inclusion

$$
\partial \sigma_{b}(T) \subseteq \partial \sigma_{b}^{*}(T) \subseteq \partial \sigma_{w}^{*}(T) \subseteq \partial \sigma_{e}^{*}(T) \subseteq \sigma_{b}^{*}(T) \subseteq \sigma_{b}(T)
$$

and hence also

$$
\sigma_{e}^{*}(T) \subseteq \sigma_{w}^{*}(T) \subseteq \sigma_{b}^{*}(T) \subseteq \sigma_{b}(T) \subseteq \eta \sigma_{e}^{*}(T)
$$


Proof. Here $\eta K$ is ([8]; [4, Definition 7.10.1]) the connected hull of a compact set $K \subseteq \mathbf{C}$, and generally ([8, Lemma 1.2, Theorem 1.3]; [4, Theorem 7.10.3]), for compact subsets $H, K \subseteq \mathbf{C}$,

$$
\partial H \subseteq K \subseteq H \Longrightarrow \partial H \subseteq \partial K \subseteq K \subseteq H \subseteq \eta K=\eta H .
$$

The analogues

$$
\sigma_{b}^{*}(T)=\sigma_{e}^{*}(T) \cup \operatorname{acc} \sigma^{*}(T)
$$

of (6.9) give equality int $\sigma^{*}(T)=\operatorname{int} \sigma_{b}^{*}(T)$ and hence

$$
\partial \sigma_{b}^{*}(T) \subseteq \partial \sigma^{*}(T),
$$

and also

$$
\sigma_{b}^{*}(T)_{\cap \text { iso }} \sigma^{*}(T) \subseteq \sigma_{e}^{*}(T),
$$

which together with the punctured neighbourhood theorems ([11, Theorem 18.7])

$$
\partial \sigma^{*}(T) \subseteq \sigma_{e}^{*}(T) \cup \text { iso } \sigma^{*}(T)
$$

give

$$
\partial \sigma_{b}^{*}(T) \subseteq \partial \sigma^{*}(T)_{\cap} \sigma_{b}^{*}(T) \subseteq \sigma_{e}^{*}(T)_{\cup}\left(\sigma_{b}^{*}(T)_{\cap \text { iso }} \sigma^{*}(T)\right)=\sigma_{e}^{*}(T) .
$$

By index continuity the sets $\sigma_{w}^{*}(T) \backslash \sigma_{e}^{*}(T)$ are all open, so that also

$$
\partial \sigma_{w}^{*}(T) \subseteq \sigma_{e}^{*}(T) \subseteq \sigma_{w}^{*}(T) .
$$

Together (10.8) and (10.9) give (10.1), and hence also (10.2).

The conditions of (8.2)-(8.5) apply to inessential operators $K \in B_{00}^{\sim}(X)$ without further commutivity assumption, which cannot more generally be relaxed, even for nilpotent operators $K \in \mathrm{Nil} B L(X)$ :

Example 11. If $S, T$ and $K$ in $B L(X)$ are defined by taking

$$
X=\left(\begin{array}{l}
E \\
E
\end{array}\right), S=\left(\begin{array}{ll}
v & 0 \\
0 & u
\end{array}\right), T=\left(\begin{array}{ll}
u & 0 \\
0 & v
\end{array}\right), K=\left(\begin{array}{ll}
0 & 1 \\
0 & 0
\end{array}\right),
$$

where $u \in B L(E)$ and $v \in B L(E)$ are the forward and the backward shifts on $E=\ell_{2}$, then $S$ and $T$ are both Weyl, while $T+K$ is not right Weyl and $S-K$ is not left Weyl.

Proof. Notice

$$
(S-K)(T+K)=I-\left(\begin{array}{cc}
0 & 0 \\
0 & 1-u v
\end{array}\right) ;(T+K)(S-K)=I-\left(\begin{array}{cc}
1-u v & 0 \\
0 & 0
\end{array}\right):
$$

both products are Fredholm of index zero, that is Weyl, and we can check that $T+K$ is one one and $S-K$ onto, with

$$
\operatorname{index}(S-K)=1=-\operatorname{index}(T+K) \text {. }
$$

Acknowledgement. We are grateful to the referee for his/her helpful comments concerning the presentation of results. 


\section{References}

[1] J. J. Buoni, R. E. Harte, and T. Wickstead, Upper and lower Fredholm spectra. I, Proc. Amer. Math. Soc. 66 (1977), no. 2, 309-314.

[2] B. P. Duggal, Perturbations of operators satisfying a local growth condition, Extracta Math. 23 (2008), no. 1, 29-42.

[3] R.E. Harte, Spectral mapping theorems, Proc. Royal Irish Acad. Sect. A 72 (1972), 89-107.

[4] , Invertibility and Singularity, Dekker 1988.

[5] - On Kato non-singularity, Studia Math. 117 (1996), no. 2, 107-114.

[6] R. E. Harte and A.-H. Kim, Weyl's theorem, tensor products and multiplication operators, J. Math. Anal. Appl. 336 (2007), no. 2, 1124-1131.

[7] R. E. Harte and W. Y. Lee, Another note on Weyl's theorem, Trans. Amer. Math. Soc. 349 (1997), no. 5, 2115-2124.

[8] R. E. Harte and A. W. Wickstead, Boundaries, hulls and spectral mapping theorems, Proc. Roy. Irish Acad. Sect. A 81 (1981), no. 2, 201-208.

[9] H. Heuser, Functional Analysis, Wiley Interscience, Chichester, 1982.

[10] J. J. Koliha, A generalized Drazin inverse, Glasgow Math. J. 38 (1996), no. 3, 367-381.

[11] V. Müller, Spectral Theory of Linear Operators and Spectral Systems in Banach Algebras, Birkhauser 2007.

[12] V. Rakočević, Essential spectrum in Banach algebras, Doctoral dissertation, Nis 1983.

[13] _ Approximate point spectrum and commuting compact perturbations, Glasgow Math. J. 28 (1986), no. 2, 193-198.

[14] _ Generalized spectrum and commuting compact perturbations, Proc. Edinburgh Math. Soc. (2) 36 (1993), no. 2, 197-209.

[15] _ Semi Browder operators and perturbations, Studia Math. 122 (1996), 131-137.

[16] M. Schechter and R. Whitley, Best Fredholm perturbation theorems, Studia Math., 90 (1988), no. 3, 175-190.

[17] S. Živković, Semi-Fredholm operators and perturbations, Inst. Math. (Beograd) (N.S.) 61(75) (1997), 73-89.

SnEŽAnA Č. ŽIvković-Zlatanović

Department of Mathematics

UNIVERSITY OF NIS

Nis 18000, SERBIA

E-mail address: mladvlad@ptt.rs

Dragan S. DJORDJEVIĆ

Department of Mathematics

UNIVERSITY OF NIS

NIS 18000, SERBIA

E-mail address: dragan@pmf.ni.ac.rs

Robin E. Harte

School of Mathematics

Trinity College Dublin

Dublin 2, IRELAND

E-mail address: rharte@maths.tcd.ie 